\title{
Impact of COVID-19 on the practice of orthopaedics and trauma-an epidemiological study of the full pandemic year of a tertiary care centre of New Delhi
}

\author{
Raju Vaishya ${ }^{1}$ (D) Abhishek Vaish ${ }^{1} \cdot$ Ashok Kumar $^{2}$ \\ Received: 26 January 2021 / Accepted: 29 March 2021 / Published online: 9 April 2021 \\ (C) SICOT aisbl 2021
}

\begin{abstract}
Purpose In an observational study, we studied the impact of COVID-19 pandemic on our clinical practice of trauma and orthopaedics, in tertiary care hospital of New Delhi.

Methods We collated the hospital data for 2019 and 2020 and analyzed and compared it extensively. We looked for the effects of the COVID-19 pandemic on several important clinical practice parameters like outpatient attendance, inpatients admissions, and surgery. The correlation of the number of surgeries done during the pandemic time was done with the number of positive cases in Delhi, monthwise. A trend of recovery was also observed.

Results During the pandemic period, the attendance of outpatients fell by $71.93 \%$, admissions by $59.35 \%$, and surgery by $55.78 \%$. Adult trauma surgery was the least affected (42.21\%), followed by arthroscopic surgery (49.81\%). Fragility hip fractures requiring bipolar hip arthroplasty were reduced by $34.15 \%$. The maximum adverse impact of the pandemic was seen on arthroplasty surgery (hip > knee), followed by on the paediatric orthopaedic cases, and spinal surgery. We notice a "lazy Vshaped" recovery after the lockdown period.

Conclusion COVID-19 pandemic has had a severe impact on all aspects of orthopaedics and trauma's clinical practice in our setup. These adverse effects were maximally seen during the lockdown period, with a reduction of $90.77 \%$ in the outpatients, $84.63 \%$ in the admissions, and $86.67 \%$ in the surgery.
\end{abstract}

Keywords COVID-19 pandemic $\cdot$ Orthopaedic surgery $\cdot$ Trauma $\cdot$ India $\cdot$ Coronavirus

\section{Introduction}

COVID-19 has impacted almost all the spheres of life, including orthopaedics and trauma [1]. The World Health Organization (WHO) announced it as a pandemic on 11 March 2020 [2]. The first case of COVID-19 in India was

Raju Vaishya

raju.vaishya@gmail.com

Abhishek Vaish

drabhishekvaish@gmail.com

Ashok Kumar

mentorashok@yahoo.com

1 Indraprastha Apollo Hospitals, Sarita Vihar, New Delhi 110076, India

2 Orthopaedic Operating Theatres, Indraprastha Apollo Hospitals, New Delhi 110076, India reported in Kerala on 30 January 2020 and in the national capital of Delhi on the 3 March 2020 [3]. As the number of cases increased to around 500, the Government of India (GOI) announced the nationwide lockdown from midnight of 24 March 2020. It was continued in four phases until 31 May 2020 (67 days).

The unlocking of restrictions was gradually done from 1 June 2020 [4]. These restrictions and increasing numbers of COVID-19 cases severely affected the healthcare delivery to the patients in orthopaedics and trauma [1, 5-7]. It also resulted in a proportionate and significant decrease in the professional income of the surgeons and hospitals $[8,9]$. Due to the initial lack of knowledge, and unpreparedness to deal with a large number of cases of this disease, a lot of confusion happened in the patient management and decision-making [10].

Although several studies have been published on the impact of COVID-19 on the practice of trauma and orthopaedics, the majority of these are based on the data of the short duration of the initial phase of pandemic and on trauma [11-13]. In this 
observational cohort study, we studied the impact of the COVID-19 pandemic on our orthopaedic and trauma practice during the lockdown, the whole pandemic period, and the entire year of 2020 and compared these with a similar time in the preceding year.

\section{Methods}

We collated the hospital data of our large multi-specialty, tertiary care corporate hospital of New Delhi, India, from our Medical Records Department for 2019 and 2020 and analyzed it extensively. We aimed to compare the impact of the COVID-19 pandemic on our practice of orthopaedic and trauma by comparing it with similar periods in the preceding year.

We looked for the effects of the COVID-19 pandemic on several important clinical practice parameters like outpatient attendance, inpatients admissions, and surgery. The correlation of the number of surgical procedures done during the pandemic time was done with the number of positive cases in Delhi, month-wise.

We divided the data into three groups:

A) Full year (from 1 January to 31 December 2020),

B) Whole pandemic period (from 25 March until 31 December 2020), and

C) Lockdown period (from 25 March to 31 May 2020).

Furthermore, the effect of the pandemic on various types of commonly performed surgery was noted under a different category of surgery like arthroplasty, arthroscopic surgery, trauma, paediatrics, spine, and miscellaneous surgery (Supplementary Table 1). A trend of recovery was also observed.

We did a thorough literature search in the first week of January 2021 in PubMed, SCOPUS, Google Scholar, and the most prominent and known orthopaedic journals' websites to find the relevant published literature for our study.

\section{Results}

The patient's attendance fell, both in the outpatients' department (Fig. 1) and for the admission and surgery (Fig. 2), in the pandemic year of 2020 (Table 1 and Fig. 3). The outpatient's attendance fell during the pandemic period of nine months and six days (a total of 281 days) by $71.93 \%$ (Table 2), and its maximum impact was during the lockdown period, where it went down to $90.77 \%$ (Table 3 ). The new and follow-up cases were reduced, with the new cases slightly more than the follow-up cases.

The admissions of orthopaedic and trauma cases went down by $59.35 \%$ in the pandemic period, and during the lockdown period (Table 3 ), the impact was maximum (84.63\%). The surgical work reduced by $55.78 \%$ during the pandemic period (Table 2) and the lockdown period by $86.67 \%$ (Table 3).

The total number of surgery done in 67 days of the lockdown was only 50 . The majority of cases done during this period were for the adult trauma (26), followed by fragility hip fractures (6), and arthroscopic surgery (6).

We noticed a steady and gradual increase in outpatient, admissions, and surgery numbers since the unlock-period, from 1 June until 31 December 2020. The number of COVID-19 cases in the city did not influence these increasing numbers of surgery (Fig. 3).

All types of orthopaedic and trauma surgery were adversely affected by the COVID-19 pandemic. Adult trauma surgery was the least affected specialty, with an overall reduction of $42.21 \%$, followed by arthroscopic surgery $(49.81 \%)$. Fragility hip fractures requiring bipolar hip arthroplasty were reduced by $34.15 \%$. The adult lower limb trauma, hip fractures, and
Fig. 1 Comparative outpatient's attendance in 2019 and 2020 (monthwise), showing a "lazy Vshaped" recovery pattern

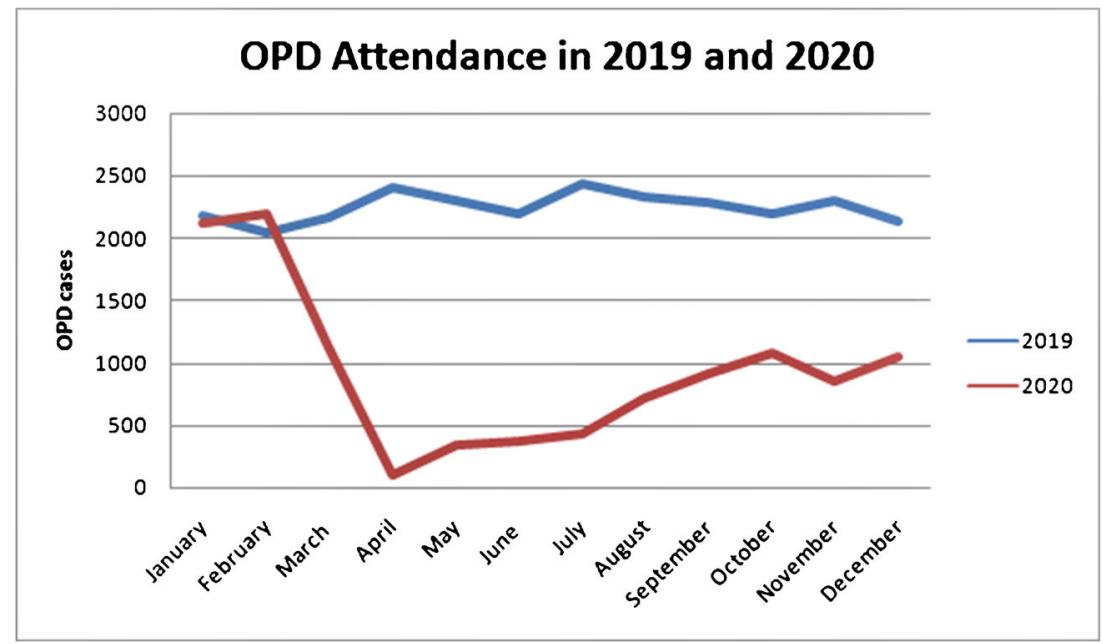


Fig. 2 Comparative numbers of surgery done in 2019 and 2020 (monthwise), showing a "lazy Vshaped" recovery pattern

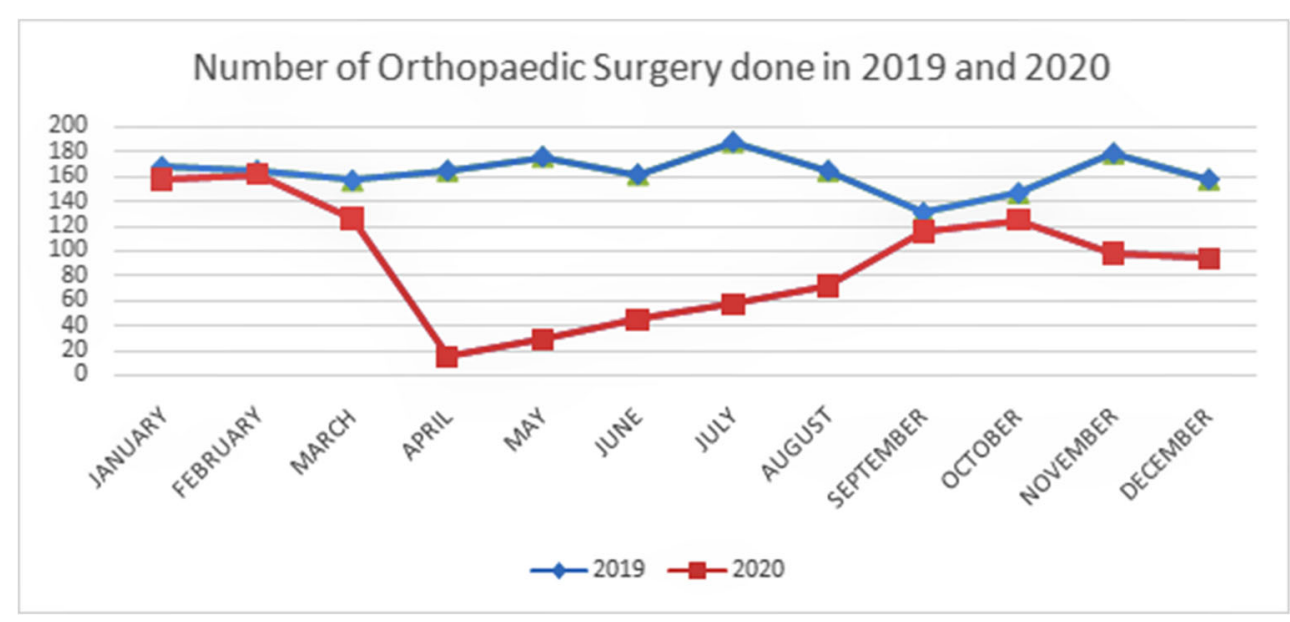

upper limb trauma were also reduced (Fig. 4). The maximum adverse impact of the pandemic was seen on arthroplasty surgery (hip $>$ knee), followed by the paediatric orthopaedic cases, and spinal surgery (Supplementary Table 1).

\section{Discussion}

COVID-19 pandemic had an immense impact on all aspects of human beings and healthcare, including trauma and orthopaedic practice, all around the world [1]. During the COVID-19 pandemic, our outpatient and surgical workloads were reduced by $71.93 \%$ and $55.78 \%$, respectively, and in the lockdown period, it was much more so with the reduction in outpatient by $90.77 \%$ and surgery by $84.63 \%$ (Fig. 5). Several previously published studies have also confirmed drastic reductions in orthopaedic practice in various parts of the world. However, these studies were mostly based on the initial impact of the pandemic during the lockdown period and mostly evaluated its effect on trauma surgery. We have however assessed the pandemic impact (for both trauma and nontrauma) for a longer time.

Table 1 A comparative pooled data of the orthopedic and trauma patients in 2019 and 2020 (group A)

\begin{tabular}{llll}
\hline Patients & 2019 & 2020 & Percentage reduction \\
\hline Outpatients & & & \\
New & 12,129 & 4816 & $60.29 \%$ \\
Follow-ups & 14,822 & 6470 & $56.34 \%$ \\
$\quad$ Total & 26,951 & 11,286 & $58.12 \%$ \\
Inpatients & & & \\
$\quad$ Admissions & 2056 & 1068 & $48.05 \%$ \\
$\quad$ Surgery & 1763 & 917 & 47.98 \\
\hline
\end{tabular}

A multicentre study from 15 centres in Italy found that all aspects of trauma care were significantly reduced: the outpatient attendance $(75 \%)$, emergency trauma $(71 \%)$, and elective surgery (up to $100 \%$ ), within the initial two weeks of the pandemic [14]. A similar trend was noticed in our centre (Fig. 5). Our orthopaedic and trauma services were maximally affected during the lockdown period. These lockdowns announced by the GOI were one of the most strict and severe lockdowns compared to any other countries in the world [4]. Still, the increasing number of COVID-19 cases in the city was not negatively correlated with the surgical cases (Fig. 3). We believe that the reasons for this dichotomy were due to the lockdown effect and there were immense fear and lack of knowledge about this disease. But later on, the needy people with trauma and other painful musculoskeletal problems started to visit the hospitals, for their treatment (mainly the surgical). Our hospital was a recognized COVID-19 center in Delhi, and hence, the face-to-face consultations were avoided by many, and here teleconsultations were used. Therefore, the outpatient attendance was more affected than the admissions and surgical work (Table 2).

Guintoli et al. from Italy also reported massive disruptions in trauma orthopaedic fracture surgeries during the COVID19 pandemic [6]. The most apparent reasons for the reduction in surgery was (a) the hospitals were aiming to save the already depleted hospital resources; (b) to decrease the risks of spreading the Coronavirus infection to the other hospital staff; (c) due to lockdown effect resulting in travel bans and forcing people to stay at home; (d) a substantial decrease in road traffic accidents, resulting in a reduction in the emergency trauma; and (e) decreased injuries related to the sporting and industrial activities [7].

In the initial part of the pandemic, there were severe confusion and a sense of "helplessness" amongst the orthopaedic surgeons, in dealing with emergency and non-emergency patients [10]. Mostly, emergency trauma and orthopaedic care were being provided during the lockdown period and initial 
Fig. 3 Correlation of monthly cases of COVID-19 in Delhi and the number of surgery done in our center

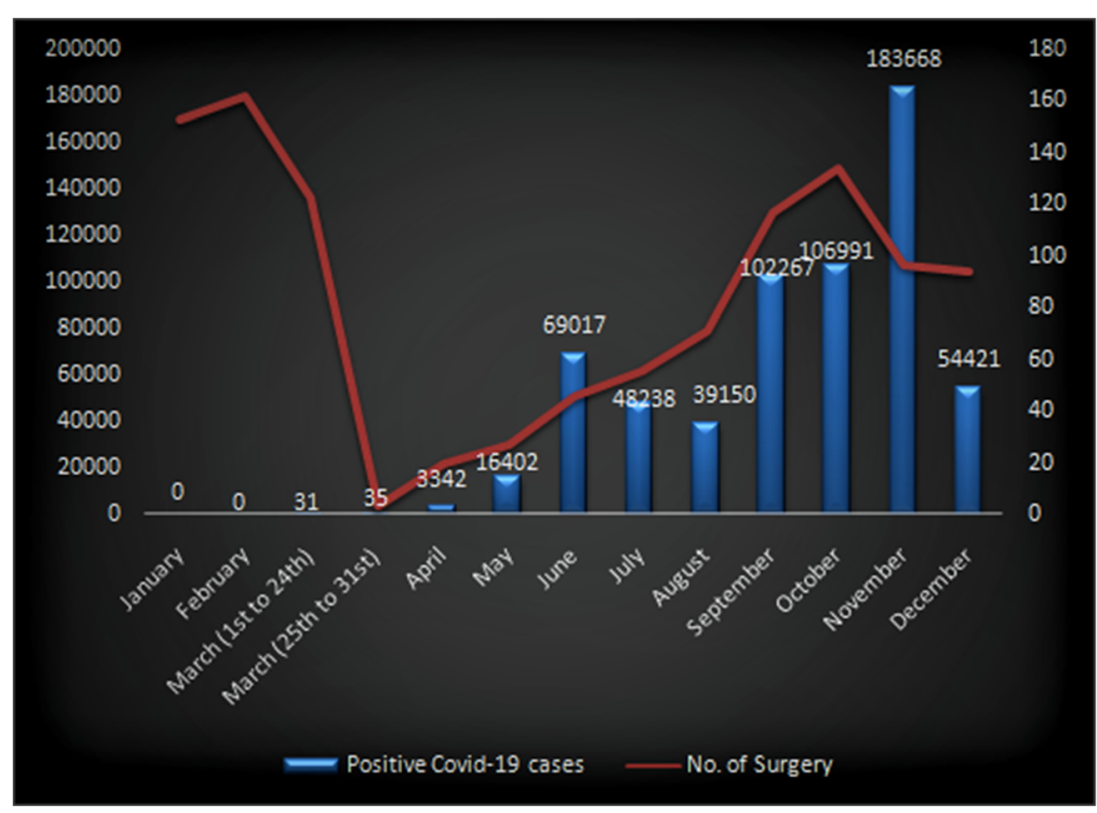

phase of the pandemic. Hence, the elective and non-emergent surgical work was maximally affected, leading to huge inconvenience to the patients and economic losses to the doctors and healthcare providers. Anoushiravani et al. [15] noticed that elective procedures accounted for $48 \%$ of hospital costs and a larger percentage of hospitals' revenues. Most hospitals suffered huge losses during the pandemic time, as the elective surgery was stopped. Battiato et al. [16] also observed a significant reduction in elective surgery nearly halved during central Italy's pandemic. Due to substantial revenue losses, many institutions had to lay off the workforce, decrease the salaries, and reduce the operating costs as much as possible, to keep themselves sustainable. Arthroplasty was affected the most in most countries, which was a similar trend as in our centre. In a retrospective study of the Medicare patients in the USA undergoing arthroplasty in the initial phase of the pandemic, there was a steep decline of 94\% (knees) and 92\% (hips), respectively [17]. Bedard et al. [18] also found a profound impact of COVID-19 on their arthroplasty volumes in the USA. Best et al. [19], in a national surgical survey, identified that all the elective orthopaedics were significantly reduced throughout the USA. This resulted in a severe loss in the incomes of the orthopaedic surgeons. They estimated the hospital losses from the cancelation of elective orthopaedic surgery during the COVID-19 pandemic were \$10.9-\$11.9 billion in reimbursement and $\$ 2.6-3.5$ billion in net income.

The negative impacts of the COVID-19 pandemic on the trauma and orthopaedic services were observed globally. Lezak et al. [20], in an online multinational survey of 63 orthopaedic trauma surgeons representing 28 countries and 14 US states, reported a significant impact of COVID-19 on $91 \%$ of participating hospitals, with only $17 \%$ of the respondents were currently performing elective orthopaedic surgery. Seventy-three percent of participating orthopaedic departments, including those in low- and middle-income countries (LMICs), had incorporated telemedicine into their practice. As a result of this event, a much-needed boost was seen in the field of telemedicine. Dhillon et al. [12] collated data from their tertiary care centre in Chandigarh, India, and evaluated their caseload during the lockdown period. They reported significant reductions in the trauma cases.

In an online survey from India, Keshav et al. [8] noticed a drastic fall in all orthopaedic practice parameters, similar to our study. They reported a fall in outpatients of over $90 \%$, and the majority of orthopaedic surgeons (64\%) had stopped elective surgery. The professional earning was reduced by more than $75 \%$, in more than $50 \%$ of respondents. The private sector surgeons were the most severely affected, while those in
Table 2 Effect of COVID-19 pandemic on the outpatient attendance, admissions, and surgery (group B)

\begin{tabular}{llll}
\hline Patients & $\begin{array}{l}\text { COVID-19 pandemic } \\
\text { period in 2020 }\end{array}$ & $\begin{array}{l}\text { Comparative } \\
\text { period of 2019 }\end{array}$ & Percentage reduction \\
\hline Outpatient's attendance & 5885 & 20972 & 71.93 \\
Admissions & 640 & 1575 & 59.35 \\
Surgery & 665 & 1504 & 55.78 \\
\hline
\end{tabular}


Table 3 Effect of lockdown on the outpatient attendance, admissions, and surgery (group C)

\begin{tabular}{llll}
\hline Patients & Lockdown period in 2020 & Comparative period of 2019 & Percentage reduction \\
\hline \multicolumn{2}{l}{ Outpatient's attendance } & & \\
New & 176 & 2281 & 92.28 \\
Follow-up & 294 & 2813 & 89.54 \\
Total & 470 & 5094 & 90.77 \\
Inpatients & & & \\
Admissions & 63 & 410 & 84.63 \\
Surgery & 50 & 375 & 86.67 \\
\hline
\end{tabular}

the government hospitals and medical colleges were the least influenced due to assured salaries. In another online survey from Germany, Haffer et al. [9] also reported substantial financial losses in Germany hospitals, with an average reduction of $49.4 \%$ in operating theatre utilization and an estimated revenue loss of $29.3 \%$, with similar reports from Scotland [21], Ireland [22], and the UK [23]. The global burden of trauma was reviewed [5], and reported a global reduction in the footfalls of trauma in the UK, Europe, Asia, the USA, Australia, and New Zealand (from 20.3 to 84.6\%). Wallace et al. [24] confirmed that the COVID-19 pandemic had an unprecedented impact on all the aspects of orthopaedic and trauma services in the UK. They also advised that in any future pandemics, the orthopaedic departments need to remain ready and flexible to infrastructural reorganization.

We believe that the epidemiological studies, like ours, provide useful information in healthcare planning and resource allocation in future pandemic situations. Although lower in the hierarchy of evidence in publications, these are valuable, as these provide direct evidence [25]. In an epidemiological study from 43 Hong Kong public hospi- tals and 122 outpatient clinics, Wong et al. [13] reported a significant drop in orthopaedic and trauma patients' attendance in the early part of the pandemic, as compared to the previous four years' similar period data. They found orthopaedic operations were reduced by $44.2 \%$, whereas elective joint replacement and ligamentous reconstruction procedures decreased by 74 to $84 \%$ ( $p<0.001)$. The number of hospital admissions was reduced by $41.2 \%$, and the outpatient attendance was reduced by $29.4 \%$. They concluded that the demand for orthopaedic care remains, despite these reductions, and advised that the orthopaedic surgeons and healthcare professionals consider staffing and resource deployment.

As the disease started to be understood over the period, the specialty work gradually increased and we have seen a "lazy V-shaped" recovery in the number of patients reporting to the outpatients and the admissions and surgery quickly (Figs. 1 and 2). We are optimistic that if the COVID-19 pandemic remains under control and with the implementation of vaccination drive, the number of patients requiring orthopaedic and trauma care for their acute and neglected problems would
Fig. 4 Comparison of the adult trauma surgery during the pandemic time, with the similar time in the preceding year

\section{Adult trauma surgery during pandemic period (25th March to 31st December 2020), compared with similar period in the preceding year}

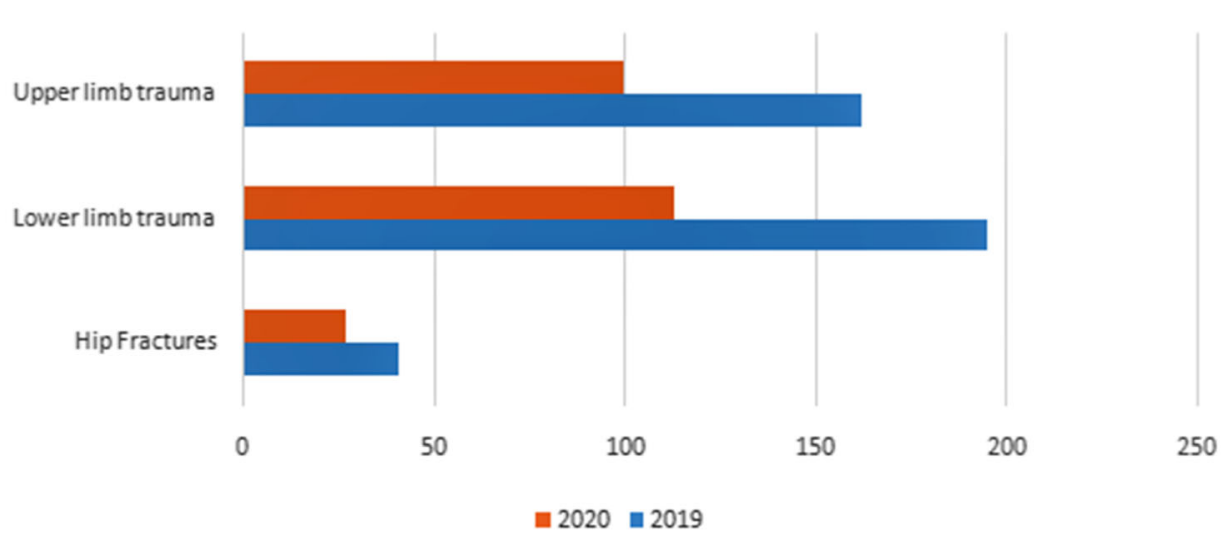


Fig. 5 Percentage reduction of the orthopaedic and trauma patients presenting to our centre in different phases of the pandemic year of 2020

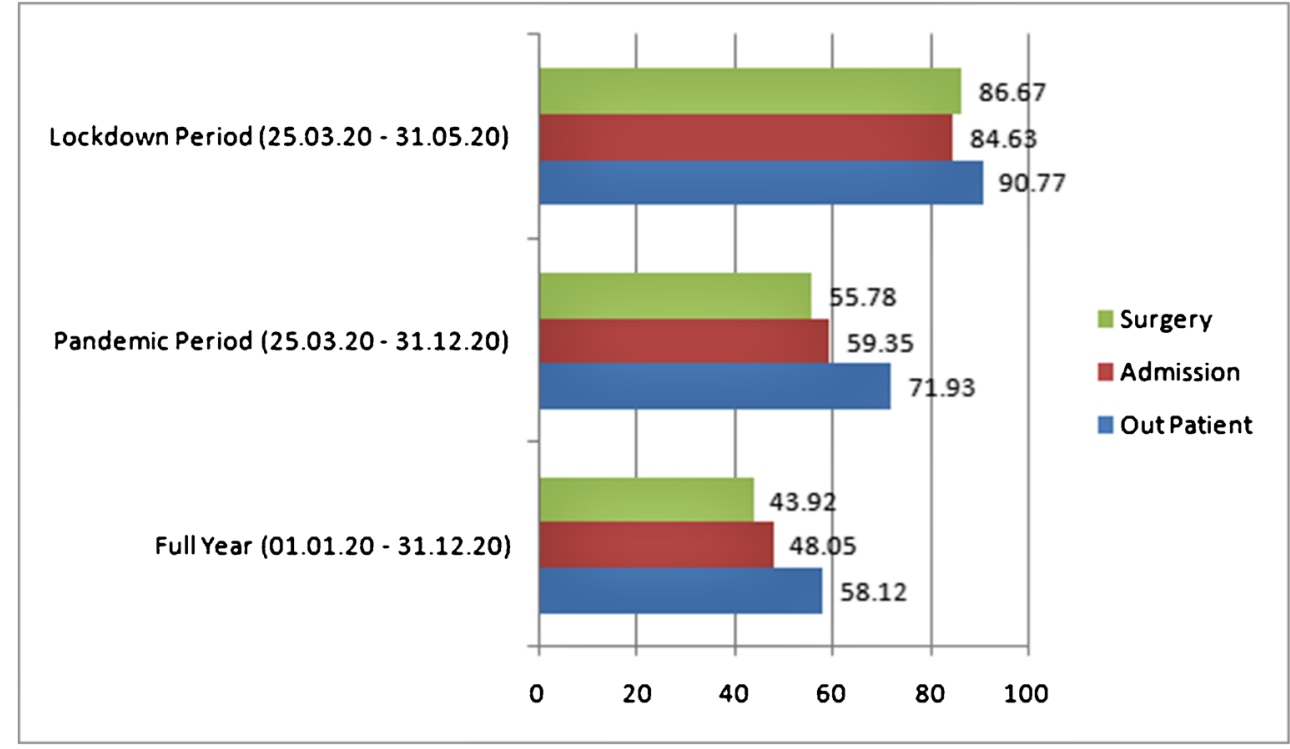

continue to rise and may overtake the numbers of the prepandemic times.

\section{Limitations of the study}

This is an epidemiological study based on a tertiary care private hospital's data. Its findings may not be fully applicable to Government institutions and the smaller hospitals, and other countries. Many essential aspects of the clinical practice of orthopaedics and traumatology were covered in this study; there may be some more included like revenue loss of the doctors and the hospital, impact on the health care providers and the patients, workforce reorganization, and hospital infrastructure which could have been covered. We suggest more multicentre study, to gain extensive data to observe the impact of the COVID-19 pandemic on all the factors related to clinical practice.

\section{Conclusion}

COVID-19 pandemic has had a severe impact on all aspects of orthopaedics and trauma's clinical practice in our setup. There was a significant reduction in the outpatients, admissions, and surgery, compared to a similar time in the preceding year. These adverse effects were maximally seen during the lockdown period, with a percentage reduction of $90.77 \%$ in the outpatient attendance, $84.63 \%$ in the hospital admissions, and $86.67 \%$ in the surgery. However, the severity of the pandemic, as seen with increasing COVID-positive cases, was not negatively correlated with the hospital work. On the contrary, the patient's numbers raised gradually since the unlocking was done, despite the disease's increasing burden in the same city. All types of orthopaedic and trauma surgery were reduced during the pandemic time, with the least impact on the adult trauma (including fragility hip fractures) and the most impact on arthroplasty. We believe that this study would provide a basis for further studies to evaluate the impact on the field of orthopaedics and traumatology. This is a unique study that has compiled the full pandemic year (2020) and compared it with the preceding year. The presented data is large enough to come to reasonable conclusions. This study should also help in resource and manpower allocation in such a human tragedy in the future.

Supplementary Information The online version contains supplementary material available at https://doi.org/10.1007/s00264-021-05021-5.

Acknowledgements We acknowledge the help of our Medical Records Department for providing us the required data for this study. Our statistician, Ms. Meenakshi Khatri, helped analyze the data and provide us the required inputs and the graphs for this paper.

Author contribution Raju Vaishya: concept framing, data analysis, manuscript writing, and editing

Abhishek Vaish: review of literature, manuscript writing, and figures Ashok Kumar: concept, data collection, and analysis

Availability of data Data are available from the Medical Records Department of the hospital.

Code availability NA

\section{Declarations}

Consent to participate NA

Consent for publication NA

Conflict of interest The authors declare no competing interests. 


\section{References}

1. Haleem A, Javaid M, Vaishya R, Vaish A (2020) Effects of COVID-19 pandemic in the field of orthopaedics. J Clin Orthop Trauma 11(3):498-499. https://doi.org/10.1016/j.jcot.2020.03.015

2. WHO announces COVID-19 outbreak a pandemic (Last accessed on $15^{\text {th }}$ January 2021). https://www.euro.who.int/en/health-topics/ health-emergencies/coronavirus-covid-19/news/news/2020/3/whoannounces-covid-19-outbreak-a-pandemic

3. COVID-19 pandemic in Delhi. Wikipedia (Last accessed on $15^{\text {th }}$ January 2021). https://en.m.wikipedia.org/wiki/COVID-19 pandemic in Delhi

4. COVID-19 lockdown in India. Wikipedia (Last accessed on 15th January 2021). https://en.wikipedia.org/wiki/COVID-19_ lockdown_in_India.

5. Waseem S, Nayar SK, Hull P, Carrothers A, Rawal J, Chou D, Khanduja V (2020) The global burden of trauma during the COVID-19 pandemic: a scoping review. J Clin Orthop Trauma. https://doi.org/10.1016/j.jcot.2020.11.005

6. Giuntoli M, Bonicoli E, Bugelli G, Valesini M, Manca M, Scaglione M (2020) Lessons learnt from COVID 19: an Italian multicentric epidemiological study of orthopaedic and trauma services. J Clin Orthop Trauma 11(4):721-727. https://doi.org/10. 1016/j.jcot.2020.05.021

7. Kumar Jain V, Lal H, Kumar Patralekh M, Vaishya R (2020) Fracture management during COVID-19 pandemic: a systematic review. J Clin Orthop Trauma 11(Suppl 4):S431-S441. https:// doi.org/10.1016/j.jcot.2020.06.035

8. Keshav K, Kumar A, Sharma P, Baghel A, Mishra P, Huda N (2020) How much has COVID-19 pandemic affected Indian orthopaedic practice? Results of an Online Survey. Indian J Orthop 54(Suppl 2):1-10. https://doi.org/10.1007/s43465-020-00218-Z

9. Haffer H, Schömig F, Rickert M, Randau T, Raschke M, Wirtz D, Pumberger M, Perka C (2020) Impact of the COVID-19 pandemic on orthopaedic and trauma surgery in university hospitals in Germany: results of a nationwide survey. J Bone Joint Surg Am 102(14):e78. https://doi.org/10.2106/JBJS.20.00756

10. Askari A, Arasteh P, Jabalameli M, Bagherifard A, Razi M (2020) COVID-19 and orthopaedic surgery: experiences from Iran. J Bone Joint Surg Am 102(13):1126-1128. https://doi.org/10.2106/JBJS. 20.00631

11. Sobti A, Memon K, Bhaskar RRP, Unnithan A, Khaleel A (2020) Outcome of trauma and orthopaedic surgery at a UK District General Hospital during the Covid-19 pandemic. J Clin Orthop Trauma 11(Suppl 4):S442-S445. https://doi.org/10.1016/j.jcot. 2020.06.042

12. Dhillon MS, Kumar D, Saini UC, Bhayana H, Gopinathan NR, Aggarwal S (2020) Changing pattern of orthopaedic trauma admissions during COVID-19 pandemic: experience at a tertiary trauma centre in India. Indian J Orthop 54(Suppl 2):1-6. https://doi.org/10. 1007/s43465-020-00241-0

13. Wong JSH, Cheung KMC (2020) Impact of COVID-19 on orthopaedic and trauma service: an epidemiological study. J Bone Joint Surg Am 102(14):e80. https://doi.org/10.2106/JBJS.20.00775
14. Benazzo F, Rossi SMP, Maniscalco P, Moretti B, Vaienti E, Ruggieri P et al (2020) The orthopaedic and traumatology scenario during Covid-19 outbreak in Italy: chronicles of a silent war. IntOrthop. 44(8):1453-1459. https://doi.org/10.1007/s00264-02004637-3

15. Anoushiravani AA, Barnes CL, Bosco JA 3rd, Bozic KJ, Huddleston JI, Kang JD, Ready JE et al (2020) Reemergence of multispecialty inpatient elective orthopaedic surgery during the COVID-19 pandemic: guidelines for a new normal. J Bone Joint Surg Am 102(14):e79. https://doi.org/10.2106/JBJS.20.00829

16. Battiato C, Berdini M, Luciani P, Gigante A (2020) Impact of coronavirus disease 2019 (COVID-19) on the epidemiology of orthopedics trauma in a region of central Italy. Injury. 51(12):29882989. https://doi.org/10.1016/j.injury.2020.09.044

17. Barnes CL, Zhang X, Stronach B, Haas DA (2021) The initial impact of COVID-19 on total hip and knee arthroplasty. J Arthroplast. https://doi.org/10.1016/j.arth.2021.01.010

18. Bedard NA, Elkins JM, Brown TS (2020) Effect of COVID-19 on hip and knee arthroplasty surgical volume in the United States. J Arthroplast 35(7S):S45-S48. https://doi.org/10.1016/j.arth.2020. 04.060

19. Best MJ, Aziz KT, McFarland EG, Anderson GF, Srikumaran U (2020) Economic implications of decreased elective orthopaedic and musculoskeletal surgery volume during the coronavirus disease 2019 pandemic. Int Orthop 44(11):2221-2228. https://doi.org/10. 1007/s00264-020-04713-8

20. Lezak BA, Cole PA Jr, Schroder LK, Cole PA (2020) Global experience of orthopaedic trauma surgeons facing COVID-19: a survey highlighting the global orthopaedic response. Int Orthop 44(8): 1519-1529. https://doi.org/10.1007/s00264-020-04644-4

21. MacDonald DRW, Neilly DW, Davies PSE, Crome CR, Jamal B, Gill SL, Jariwala AC et al (2020) Effects of the COVID-19 lockdown on orthopedic trauma: a multicentre study across Scotland. Bone Jt Open. 1(9):541-548. https://doi.org/10.1302/2633-1462.19

22. Fahy S, Moore J, Kelly M, Flannery O, Kenny P (2020) Analysing the variation in volume and nature of trauma presentations during COVID-19 lockdown in Ireland. Bone Jt Open 1(6):261-266. https://doi.org/10.1302/2046-3758.16

23. Murphy T, Akehurst H, Mutimer J (2020) Impact of the 2020 COVID-19 pandemic on the workload of the orthopedic service in a busy UK district general hospital. Injury. 51(10):2142-2147. https://doi.org/10.1016/j.injury.2020.07.001

24. Wallace CN, Kontoghiorghe C, Kayani B, Chang JS, Haddad FS (2020) The impact of COVID-19 on trauma and orthopedic surgery in the United Kingdom. Bone Jt Open. 1(7):420-423. https://doi. org $/ 10.1302 / 2633-1462.17$

25. Calderon RL (2000) Measuring risks in humans: the promise and practice of epidemiology. Food Chem Toxicol 38(1 Suppl):S59 S63. https://doi.org/10.1016/s0278-6915(99)00134-9

Publisher's note Springer Nature remains neutral with regard to jurisdictional claims in published maps and institutional affiliations. 